\title{
Roles of P-glycoprotein and multidrug resistance protein in transporting para-aminosalicylic acid and its $\mathbf{N}$-acetylated metabolite in mice brain
}

\author{
Lan HONG ${ }^{1,2}$, Cong XU1 ${ }^{1}$, Stefanie O'NEAL ${ }^{2}$, Hui-chang $\mathrm{BI}^{3}$, Min HUANG ${ }^{3}$, Wei ZHENG ${ }^{2, *}$, Su ZENG ${ }^{1, *}$ \\ ${ }^{1}$ College of Pharmaceutical Sciences, Zhejiang Province Key Laboratory of Anti-Cancer Drug Research, Zhejiang University, Hangzhou \\ 310058, China; ${ }^{2}$ School of Health Sciences, Purdue University, West Lafayette, Indiana, USA; ${ }^{3}$ Institute of Clinical Pharmacology, \\ School of Pharmaceutical Sciences, Sun Yat-sen University, Guangzhou 510006, China
}

Aim: Para-aminosalicylic acid (PAS) is effective in the treatment of manganism-induced neurotoxicity (manganism). In this study we investigated the roles of P-glycoprotein (MDR1a) and multidrug resistance protein (MRP) in transporting PAS and its $N$-acetylated metabolite AcPAS through blood-brain barrier.

Methods: MDR1a-null or wild-type mice were intravenously injected with PAS (200 mg/kg). Thirty minutes after the injection, blood samples and brains were collected, and the concentrations of PAS and AcPAS in brain capillaries and parenchyma were measured using HPLC. Both MDCK-MDR1 and MDCK-MRP1 cells that overexpressed P-gp and MRP1, respectively, were used in two-chamber Transwell transport studies in vitro.

Results: After injection of PAS, the brain concentration of PAS was substantially higher in MDR1a-null mice than in wild-type mice, but the brain concentration of AcPAS had no significant difference between MDR1a-null mice and wild-type mice. Concomitant injection of PAS with the MRP-specific inhibitor MK-571 (50 mg/kg) further increased the brain concentration of PAS in MDR1a-null mice, and increased the brain concentration of AcPAS in both MDR1a-null mice and wild-type mice. Two-chamber Transwell studies with MDCKMDR1 cells demonstrated that PAS was not only a substrate but also a competitive inhibitor of P-gp, while AcPAS was not a substrate of P-gp. Two-chamber Transwell studies with the MDCK-MRP1 cells showed that MRP1 had the ability to transport both PAS and AcPAS across the BBB.

Conclusion: P-gp plays a major role in the efflux of PAS from brain parenchyma into blood in mice, while MRP1 is involved in both PAS and AcPAS transport in the brain.

Keywords: para-aminosalicylic acid; manganism; chelating agent; blood-brain barrier; MK-571; P-glycoprotein; multidrug resistance protein; MDCK-MDR1 cells; MDCK-MRP1 cells

Acta Pharmacologica Sinica (2014) 35: 1577-1585; doi: 10.1038/aps.2014.103; published online Nov 242014

\section{Introduction}

Excessive exposure to manganese (Mn) can lead to a Mninduced neurotoxicity that is clinically called manganism and has signs and symptoms similar to, but not identical to Parkinson's disease ${ }^{[1-4]}$. The mechanism of Mn-induced neurotoxicity remains elusive and clinical interventions are limited ${ }^{[5-8]}$. Chelation therapy with calcium sodium ethylenediaminetetraacetate acid ( $\mathrm{CaNa}_{2}$ EDTA) has been suggested as a strategy to reduce the body burden of $\mathrm{Mn}^{[3,9]}$. Yet, the CaNa ${ }_{2}$ EDTA therapy is not effective because of the limited permeability of

\footnotetext{
* To whom correspondence should be addressed.

E-mail zengsu@zju.edu.cn (Su ZENG); wzheng@purdue.edu (Wei ZHENG)

Received 2014-06-21 Accepted 2014-09-02
}

$\mathrm{CaNa}_{2} \mathrm{EDTA}_{\mathrm{A}}$ across the blood-brain barrier ${ }^{[8]}$. Therefore, there is a pressing need to discover an effective therapeutic agent for the treatment of manganism.

Para-aminosalicylic acid (PAS) is a well-established, antituberculosis drug since $1959^{[10]}$. Reports in the literature have shown its effectiveness in the treatment of Mn-induced neurotoxicity ${ }^{[11-17]}$. Evidence has also been provided regarding the effective removal of $\mathrm{Mn}$ from the CSF and brain tissues in rats following PAS treatment ${ }^{[18]}$. More recently, Santos and colleagues reported that PAS is effective in reducing brain Mn burden ${ }^{[19,20]}$. The authors further suggest that in addition to its role in metal chelation, the anti-inflammatory property of PAS allows it to reduce the level of prostaglandin $\mathrm{E}_{2}\left(\mathrm{PGE}_{2}\right)$, which is crucial for the development of neuroinflammation ${ }^{[19]}$.

To understand the mechanism by which PAS alleviates the 
Mn-induced parkinsonian syndromes, we have used in vivo methods to quantify the regional pharmacokinetics of PAS and its major metabolite, $N$-acetyl-para-aminosalicylic acid (AcPAS), in the rat brain ${ }^{[21]}$. Our data show that both PAS and AcPAS possess the ability to enter the brain parenchyma, but AcPAS has a much higher brain concentration and longer $t_{1 / 2}$ than PAS. It is known that the chemical distribution in the brain is influenced by the brain barrier systems ${ }^{[22]}$. The tight junctions between the brain capillary endothelial cells form the blood-brain barrier (BBB), whereas the tight junctions between the choroid plexus epithelial cells constitute the blood-CSF barrier $(\mathrm{BCB})^{[23-25]}$. The lower PAS brain concentration could be due to the greater transport of PAS back to the blood by the efflux-transport systems present at the BBB.

Multidrug resistance protein 1 (MDR1), also called P-glycoprotein (P-gp) or ATP-binding cassette subfamily B member 1 (ABCB1), is an efflux transport system that selectively transports substrates from the interstitial fluid (ISF) to the blood and therefore restricts the entry of materials from the blood into the brain parenchyma ${ }^{[26]}$. In addition, the multidrug resistance-associated protein 1 (MRP1), a member of the superfamily of ATP-binding cassette (ABC) transporters, is known to express at the basolateral side of the choroid plexus epithelial cells and functions to transport materials from the CSF to the blood $^{[26-31]}$. We hypothesize that both P-gp and MRP1 play an important role in transporting PAS and AcPAS between the blood and the brain.

The purposes of this study were to investigate (1) whether PAS and AcPAS are the substrates of two well-defined drug transporters, ie, P-gp and MRP1, and (2) whether these two efflux transporters mediated the efflux of PAS and/or AcPAS in the BBB. The Mdr1a-null mice and a specific MRP inhibitor MK-571 were used in animal studies in vivo. MDCK-MDR1 and MDCK-MRP1 transfected cells overexpressing P-gp and MRP1, respectively, are recommended by the US FDA as valuable tools for the prediction of P-gp and MRP1 substrates ${ }^{[32]}$. These two transfected cell lines were used in our two-chamber Transwell transport studies in vitro.

\section{Materials and methods Chemicals}

PAS, dimethyl sulfoxide (DMSO), 3-(4,5-dimethyl-2-thiazolyl)2,5-diphenyl-2H-tetrazolium bromide (MTT), phenol red, rhodamine 123 (R123), MK-571, verapamil, quinidine and calcein-AM were purchased from Sigma-Aldrich (Saint Louis, MO, USA); high-glucose Dulbecco's modified Eagle's medium (DMEM) was purchased from Invitrogen Life Technologies Inc (Carlsbad, CA, USA); and fetal bovine serum was purchased from Gibco Co (Bethesda, MD, USA). AcPAS was synthesized in this laboratory ${ }^{[33]}$. HPLC-grade water was prepared using the NANOpure Diamond Ultrapure Water Systems (Barnstead International, Dubuque, IA, USA). All of the reagents were of analytical grade, HPLC grade, or the best available pharmaceutical grade.

\section{Animals and treatments}

Male, 8-week-old MDR1a-deficient mice and age-matched male wild-type (WT) mice were purchased from Charles River Laboratories, Inc (Wilmington, MA, USA) and weighed 25 $\pm 1 \mathrm{~g}$ (mean $\pm S D$ ) at the time of experimentation. Animals were acclimatized for 1 week prior to experimentation in a temperature-controlled, 12/12-h light/dark room and were allowed free access to deionized water and rodent feed. The housing facility at Purdue University is accredited by the Association for Assessment and Accreditation of Laboratory Animal Care. Before experimentation, the animals were fasted for $12 \mathrm{~h}$ with free access to water. The animal treatment protocol was approved by the Institutional Committee on Animal Use at Purdue University.

PAS was dissolved in sterile saline each day prior to administration. PAS (200 mg/ kg) was injected intravenously (iv) in the tail vein. For the PAS and MK-571 treatment group, PAS (200 mg/kg) was mixed with MK-571 (50 mg/kg) and ivinjected via the tail vein as described previously ${ }^{[21,33]}$. Thirty minutes after administration, the mice were anesthetized, and blood samples were collected by heart puncture. The brain was rapidly dissected from the skull, washed with ice-cold saline, and dissected mid-sagittally. One hemisphere was used for the brain regional capillaries isolation study. The capillary depletion process was performed according to a well-established procedure in this laboratory ${ }^{[33,34]}$. The other hemisphere was homogenized using a homogenization buffer.

\section{HPLC analysis}

The concentrations of PAS and AcPAS in plasma and brain samples were quantified using a well-established HPLC method developed in this laboratory ${ }^{[33]}$. The presence of transporter inhibitors in samples did not interfere with the HPLC quantification of PAS or AcPAS.

\section{Cell culture}

The MDCK-transfected MDCK-MDR1 cell line was developed in this laboratory ${ }^{[35]}$. The MRP1-transfected MDCK-MRP1 cell line was a gift from Dr P BORST (Netherlands Cancer Institute, Amsterdam, The Netherlands). MDCK cells were cultured in DMEM supplemented with 10\% FBS and 1\% nonessential amino acid. Cells were grown at $37^{\circ} \mathrm{C}$ in a humidified atmosphere of $5 \% \mathrm{CO}_{2}$ in the air.

\section{Cytotoxicity assay}

Cytotoxicity was measured using the MTT assay in 96-well plates. After reaching $80 \%$ confluency, the cells were treated with PAS (430 and $2 \mu \mathrm{g} / \mathrm{mL}$ ) or AcPAS (50 and $2 \mu \mathrm{g} / \mathrm{mL}$ ) for $48 \mathrm{~h}$. The concentrations of PAS or AcPAS used in the cell cultures were selected based on the plasma concentration of both chemicals after PAS iv administration ${ }^{[21,33]}$. At the end of the 48-h treatment, the cells were washed twice with PBS and incubated with $150 \mu \mathrm{L}$ of PBS-MTT $(2.0 \mathrm{mg} / \mathrm{mL})$ at $37^{\circ} \mathrm{C}$ for $4 \mathrm{~h}$. The supernatant was carefully removed, followed by the addition of $150 \mu \mathrm{L}$ of DMSO. The formazan product was 
quantified at $590 \mathrm{~nm}$ with a microplate reader (SpectraMax M2, Molecular Devices, Sunnyvale, CA, USA).

\section{Cell-based Transwell transport study}

Cells were cultured in the inner chamber of the Transwell apparatus with a seeding density of $2 \times 10^{5}$ cells $/ \mathrm{cm}^{2}$; the inner chamber was inserted in the outer chamber and cultured for $5 \mathrm{~d}$ prior to the transport studies. The media was changed every other day for the first $4 \mathrm{~d}$ and then every day thereafter. The transepithelial electrical resistance (TEER), an indicator of the tightness of the barrier, was recorded to track the formation of the monolayer tight barrier between the inner and the outer chambers. Phenol red was used as a reference leakage marker to conduct bi-directional transport studies in the Transwells. No interaction between phenol red and either PAS or AcPAS was found in our preliminary study. The transport experiments were conducted in Hank's Balanced Stock Solutions (HBSS) at $37^{\circ} \mathrm{C}$. PAS, AcPAS and phenol red permeability values were calculated from the linear portion of the uptake curves. Apparent permeability $\left(P_{\text {app }}\right)$ was calculated for the cell monolayer on filters and for the empty filters as follows:

$$
P_{\text {app }}=V_{\text {Receiver }} /\left(A_{\text {filter }} \times C_{\text {Donor }}\right) \times \delta C_{\text {Receiver }} / \delta \mathrm{T}
$$

where $V_{\text {Receiver }}$ is the volume of the receiver chamber, $A_{\text {filter }}$ is the filter surface area, $C_{\text {Donor }}$ is the initial concentration in the donor chamber, and $C_{\text {Receiver }} / \delta \mathrm{T}$ is the change in the receiver chamber concentration over time. Monolayer permeability $\left(P_{\mathrm{E}}\right)$ was determined from the $P_{\text {app }}$ values of the cells and the blank filters using the following equation:

$$
1 / P_{\mathrm{E}}=1 / P_{\text {cells }}-1 / P_{\text {blank }}
$$

\section{Transport study of PAS and AcPAS in MDCK-MDR1 cells}

In theory, the MDCK cells cultured on the membrane of the inner chamber directly contacted the "blood". Thus, to study the efflux transport of PAS or AcPAS at the BBB (ie, from the CSF to the blood), PAS or AcPAS was added to the outer chamber and their concentrations in the inner chamber were determined. The final concentrations in the outer chamber were 430 and $2 \mu \mathrm{g} / \mathrm{mL}$ of PAS for the high- and low-concentration groups, respectively, or 50 and $2 \mu \mathrm{g} / \mathrm{mL}$ of AcPAS for the high- and low-concentration groups, respectively. The final concentration of phenol red was $20 \mu \mathrm{g} / \mathrm{mL}$. At $0,20,40$, $60,80,100$, and $120 \mathrm{~min}$, aliquots $(100 \mu \mathrm{L})$ of culture media were taken from the inner (receiver) chamber and replaced with $100 \mu \mathrm{L}$ of pre-warmed HBSS (to keep constant hydrostatic pressure during the entire experiment). To examine the influx transport of PAS or AcPAS (ie, from the inner to the outer chamber), PAS or AcPAS was added into the inner chamber and aliquots $(100 \mu \mathrm{L})$ of culture media were taken from the outer (receiver) chamber with replacement of $100 \mu \mathrm{L}$ of pre-warmed HBSS.

R123 is a known substrate of P-gp and was used as a positive control to study P-gp-mediated efflux. R123 was added to the chambers with a final concentration of $5 \mu \mathrm{mol} / \mathrm{L}$ and quantified at an excitation wavelength of $485 \mathrm{~nm}$ and an emission wavelength of $535 \mathrm{~nm}$ using a freshly prepared standard curve (6 samples, 0.005 to $0.5 \mu \mathrm{g} / \mathrm{mL}$, linearity: $r=0.9996)$. The P-gp inhibitors verapamil and quinidine were prepared in DMSO and added to the cultured cells at a final concentration of $50 \mu \mathrm{mol} / \mathrm{L}$. The final concentration of the solvent DMSO was less than $0.1 \%$ in all of the experiments. The same volume of DMSO was added to the transport buffer as a negative control. All of the samples were placed in Eppendorf tubes and immediately stored at $4{ }^{\circ} \mathrm{C}$ until HPLC analysis. The net efflux ratio (NER) was determined by calculating the ratio of the efflux ratio (ER) value from the MDCK-MDR1 cell studies divided by the ER value from the MDCK wild-type cell studies.

To investigate the inhibitory effect of PAS on the activity of P-gp at MDCK-MDR1 cells, various concentrations of freshly prepared PAS $(0,1.0,5.0,10.0,20.0,50.0,100$, and $400 \mu \mathrm{g} / \mathrm{mL})$ were used to determine the $\mathrm{IC}_{50}$ from dose-response curves.

\section{Transport study of PAS and AcPAS in MDCK-MRP1 cells}

The experiments were conducted in the same manner as described above. Calcein-AM is a known MRP1 substrate and was used as a positive control to study MRP1-mediated efflux. Calcein-AM was added to a final concentration of $4 \mu \mathrm{mol} / \mathrm{L}$ and was quantified at an excitation wavelength of $485 \mathrm{~nm}$ and an emission wavelength of $525 \mathrm{~nm}$ from the calcein-AM standard curve ( 6 samples, 0.005 to $1 \mu \mathrm{g} / \mathrm{mL}$ ). The MRP1 inhibitor MK-571 $(20 \mu \mathrm{mol} / \mathrm{L})$ was prepared in DMSO. The same volume of DMSO was added as a negative control.

\section{Statistical analysis}

All data are presented as the mean \pm SD. Statistical analysis for the comparison of two means was performed using one-way ANOVA. In all cases, a probability level of $P$-value $<0.05$ was considered as the criterion for significance.

\section{Results}

Involvement of P-gp in the brain distribution of PAS and AcPAS in vivo

To understand if P-gp is involved in the transport of PAS and AcPAS, comparisons of the brain tissue/plasma $(\mathrm{T} / \mathrm{P})$ ratios of these two molecules were made between genetically MDR1adeficient and wild-type mice. Brain and plasma samples were collected from the MDR1a-deficient and wild-type mice $30 \mathrm{~min}$ after iv injection; this time point was chosen because our previous pharmacokinetic studies revealed that the $t_{\max }$ of both PAS and AcPAS was approximately $30 \mathrm{~min}$ after PAS injection ${ }^{[21]}$. The brain capillary fraction forms the BBB and by examining the level of PAS in the brain capillary we could know the concentration of PAS in the BBB. Following the iv injection of PAS, the concentrations of the metabolite AcPAS in the brain parenchyma and capillary fractions were generally higher than those of PAS, an observation that substantiated our previous report ${ }^{[21,33]}$. PAS concentrations in the brain parenchyma were significantly higher in the MDR1a-deficient mice (6.66 $\mu \mathrm{g} / \mathrm{g})$ than in the wild-type mice $(1.80 \mu \mathrm{g} / \mathrm{g})(P<0.05)$; coincidently, PAS concentrations in the brain capillaries were also much greater in the MDR1a-deficient mice $(3.35 \mu \mathrm{g} / \mathrm{g})$ than in 
Table 1. Concentrations of PAS and AcPAS in the brain capillary and parenchyma of wild-type and MDR1a-deficient mice $(\mu \mathrm{g} / \mathrm{g})$. Data represent the mean \pm SD. $n=3 .{ }^{b} P<0.05$ compared to the wild-type mice.

\begin{tabular}{llll}
\hline Analyte & \multicolumn{1}{c}{ Tissue } & Wild-type & MDR1a-deficient \\
\hline PAS & Brain capillary & $0.162 \pm 0.050$ & $3.347 \pm 0.686^{\mathrm{b}}$ \\
& Brain parenchyma & $1.798 \pm 0.694$ & $6.662 \pm 0.965^{\mathrm{b}}$ \\
& Plasma & $179.4 \pm 14.90$ & $328.1 \pm 40.33^{\mathrm{b}}$ \\
& & & \\
AcPAS & Brain capillary & $1.278 \pm 0.446$ & $1.546 \pm 0.414$ \\
& Brain parenchyma & $4.762 \pm 0.985$ & $4.030 \pm 0.690$ \\
& Plasma & $43.37 \pm 14.60$ & $53.50 \pm 11.99$ \\
\hline
\end{tabular}

the wild-type mice $(0.16 \mu \mathrm{g} / \mathrm{g})(P<0.05)$ (Table 1$)$. The data in Figure 1 indicate that the PAS tissue-to-plasma ratios $(\mathrm{T} / \mathrm{P})$ in the brain capillary and the parenchyma were 11.3-fold and 2.0fold, respectively, greater in the transgenic mice than in the wild-type mice, suggesting a P-gp-associated brain accumulation of PAS.

Unlike PAS, there was no statistically significant difference in the AcPAS concentration or $\mathrm{T} / \mathrm{P}$ ratio between the MDR1adeficient and the wild-type mice (Table 1, Figure 1), suggesting that P-gp plays a less important role in AcPAS brain distribution.

Involvement of MRP in PAS and AcPAS brain distribution in vivo In the wild-type animals treated with the MRP inhibitor MK-571, the treatment did not significantly affect the PAS $\mathrm{T} / \mathrm{P}$ ratio; however, in the MDR1a-deficient mice, treatment with MK-571 further increased the PAS T/P ratio in the brain

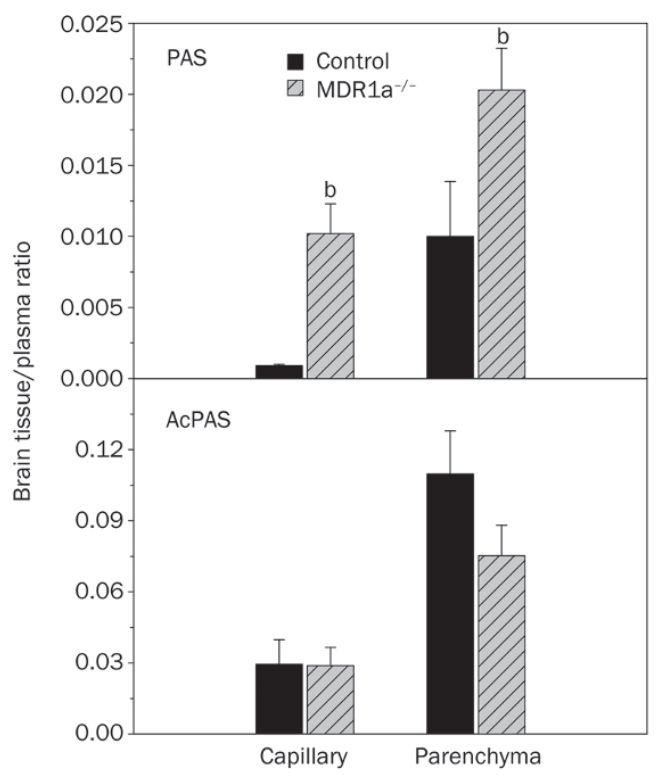

Figure 1. T/P ratio of PAS and AcPAS in the brain parenchyma and capillary. MDR1a-null and wild-type mice received $200 \mathrm{mg} / \mathrm{kg}$ of PAS via iv injection through the tail vein. At 30 min after administration, the mice were sacrificed. Concentrations of PAS and AcPAS in plasma and brain samples were quantified by HPLC. Data represent the mean \pm SD. $n=3$. ${ }^{\mathrm{b}} P<0.05$ compared to wild-type mice.

parenchyma as well as in the brain capillary (Figure 2). Unlike PAS, treatment of the wild-type mice with MK-571 significantly increased the AcPAS T/P ratios in the brain capillary by 1.8 -fold and in the brain parenchyma by 1.5 -fold $(P<0.05)$ (Figure 2). While there was no statistically significant differ-
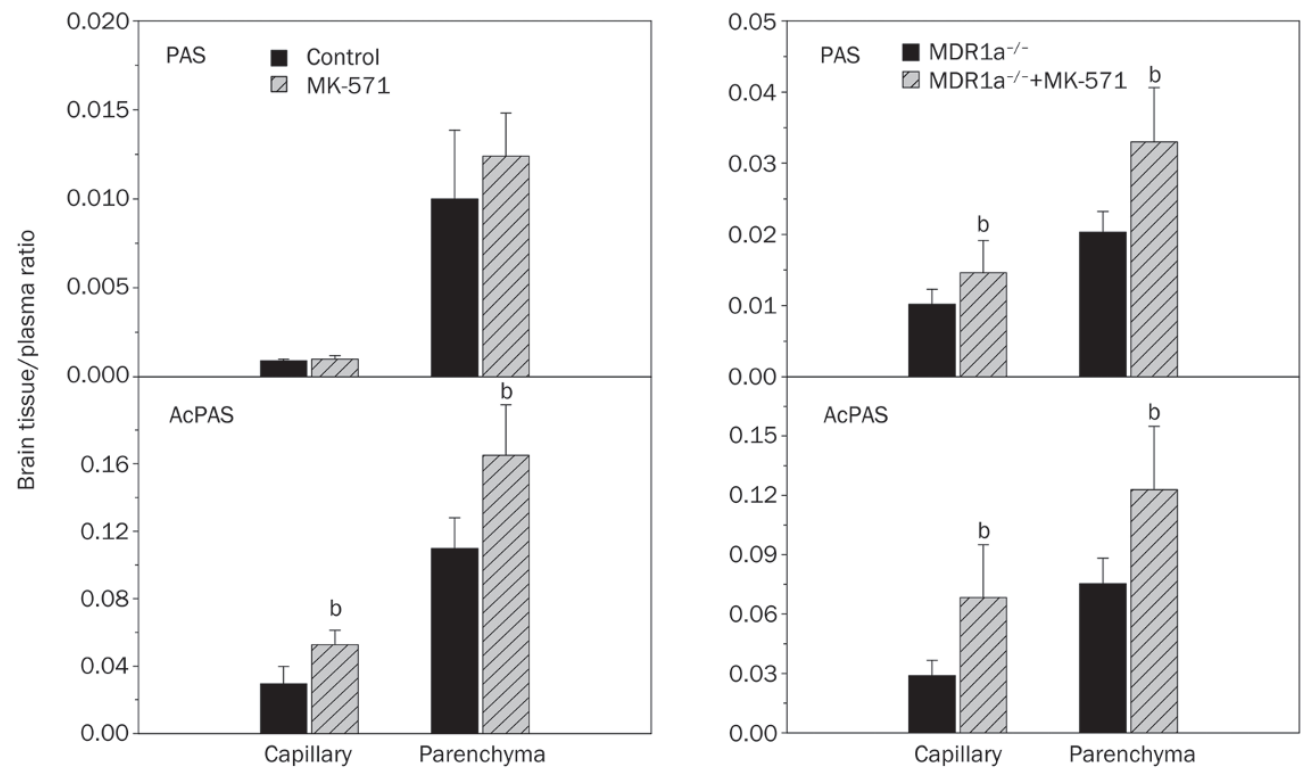

Figure 2. T/P ratios of PAS and AcPAS in the brain parenchyma and capillary. MDR1a-null and wild-type mice received $200 \mathrm{mg} / \mathrm{kg}$ of PAS via iv injection through the tail vein. For the MK-571 treatment group, PAS (200 mg/kg) was mixed with MK-571 (50 mg/kg) prior to injection. Data represent the mean \pm SD. $n=3 .{ }^{b} P<0.05$ compared to the group not given MK-571. 
ence in AcPAS T/P ratio between the MDR1a-deficient and the wild-type mice (Figure 1), the injection of MK-571 in the MDR1a-deficient mice resulted in significantly higher AcPAS $\mathrm{T} / \mathrm{P}$ ratios in the brain parenchyma and capillary than without MRP inhibition (Figure 2). These observations suggest that the MRP were involved in the PAS and AcPAS efflux at the brain barrier.

\section{Cytotoxicity assay}

The cytotoxicity of PAS and AcPAS for all of the different cell lines was examined by MTT assays, using a $0.1 \%$ Triton X-100 solution as a positive control and a DMEM solution as a negative control. Cells were treated with PAS (430 and $2 \mu \mathrm{g} / \mathrm{mL}$ ) or AcPAS (50 and $2 \mu \mathrm{g} / \mathrm{mL}$ ) at the high and low concentrations, respectively, for $48 \mathrm{~h}$. No significant changes in cell viability were observed in these experiments (data not shown). Thus, all of the following cell transport studies were performed with non-cytotoxic PAS or AcPAS concentrations.

\section{Transport study of PAS and AcPAS in MDCK-MDR1 cells}

MDCK-MDR1 cells were used as the in vitro model to mimic the P-gp-mediated drug transport at the BBB. After the cells were cultured on the inner chamber for $60 \mathrm{~h}$, the TEER values reached above $600 \Omega \cdot \mathrm{cm}^{2}$, and the $P_{\mathrm{E}}$ value of the paracellular space marker phenol red was below $2.44 \times 10^{-8} \mathrm{~cm} / \mathrm{s}$, which suggested the formation of the tight barrier between the two chambers. The concentration-time curves of both compounds were linear in both the efflux and the influx studies up to $2 \mathrm{~h}$ (data not shown). The PAS $P_{\mathrm{E}}$ values of the wild-type MDCK cell monolayer (MDCK-WT) and the MDCK-MDR1 cell monolayer were below $1 \times 10^{-6} \mathrm{~cm} / \mathrm{s}$, showing a low capability of $\mathrm{PAS}$ to cross the BBB. The PAS net efflux ratio (NER) transport in the MDCK-MDR1/MDCK-WT cell monolayer at the high concentration was 1.05, while the PAS NER at the low concentration was 3.80. For the positive control R123, the net efflux ratio was 6.10 (Table 2). According to the FDA recommendations, a compound with an NER above 2.0 is considered to be a typical substrate of $\mathrm{P}-\mathrm{g} \mathrm{p}^{[32]}$.

When either the P-gp inhibitor verapamil (50 $\mu \mathrm{mol} / \mathrm{L})$ or quinidine $(50 \mu \mathrm{mol} / \mathrm{L})$ was incubated with the MDCK-MDR1

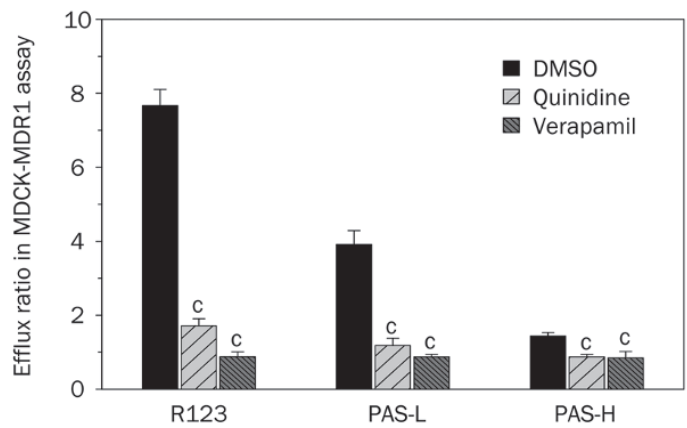

Figure 3. Effects of the P-gp inhibitors quinidine $(50 \mu \mathrm{mol} / \mathrm{L})$ and verapamil $(50 \mu \mathrm{mol} / \mathrm{L})$ on the efflux ratio of R123 and PAS across the MDCK-MDR1 cell monolayer. DMSO was used as the negative control. The PAS-L group received $2 \mu \mathrm{g} / \mathrm{mL}$ of PAS in the culture media and served as the low concentration group. The PAS-H group received $430 \mu \mathrm{g} / \mathrm{mL}$ of PAS in the culture media and served as the high concentration group. Data represent the mean \pm SD. $n=3 .{ }^{c} P<0.01$ compared to controls.

cells, the efflux of R123 and PAS at the MDCK-MDR1 cell monolayer was significantly blocked (Figure 3), indicating that PAS was a potential P-gp substrate. The AcPAS NER at both the high and low concentration levels were below 1.0 (Table 2).

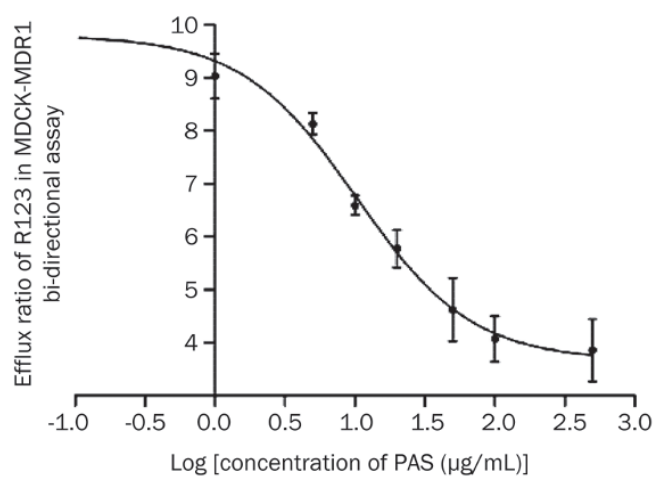

Figure 4. Efflux ratio of R123 across the MDR1-MDCK cell monolayer in the presence of various concentrations of PAS. Data represent the mean \pm SD. $n=3$. The $I C_{50}$ was estimated to be $10.29 \pm 3.52 \mu \mathrm{g} / \mathrm{mL}$.

Table 2. Permeability $\left(P_{\mathrm{E}}\right)$ of PAS and AcPAS through the MDCK/MDCK-MDR1 cell monolayer in the Transwell devices. Data represent the mean \pm SD. $n=3$.

\begin{tabular}{|c|c|c|c|c|c|c|}
\hline & \multirow{2}{*}{$\begin{array}{l}\text { Permeability } \\
\left(\times 10^{-7} \mathrm{~cm} / \mathrm{s}\right)\end{array}$} & \multicolumn{2}{|c|}{ MDCK monolayer } & \multicolumn{2}{|c|}{ MDCK-MDR1 monolayer } & \multirow{2}{*}{$\begin{array}{l}\text { Net efflux } \\
\text { ratio (NER) }\end{array}$} \\
\hline & & Influx & Efflux & Influx & Efflux & \\
\hline \multirow[t]{3}{*}{ PAS } & R123 (5 mol/L) & $2.825 \pm 0.867$ & $5.590 \pm 0.435$ & $2.494 \pm 0.077$ & $30.120 \pm 0.924$ & 6.103 \\
\hline & High conc $(430 \mu \mathrm{g} / \mathrm{mL})$ & $7.202 \pm 0.198$ & $5.065 \pm 0.194$ & $7.125 \pm 0.442$ & $5.279 \pm 0.346$ & 1.054 \\
\hline & Low conc $(2 \mu \mathrm{g} / \mathrm{mL})$ & $3.685 \pm 0.290$ & $2.546 \pm 0.298$ & $1.194 \pm 0.076$ & $3.132 \pm 0.279$ & 3.796 \\
\hline \multirow[t]{2}{*}{ AcPAS } & High conc (50 g/mL) & $31.04 \pm 0.140$ & $22.51 \pm 0.097$ & $24.24 \pm 0.182$ & $17.20 \pm 0.076$ & 0.977 \\
\hline & Low conc $(2 \mu \mathrm{g} / \mathrm{mL})$ & $48.93 \pm 0.881$ & $34.89 \pm 1.411$ & $20.46 \pm 1.805$ & $12.69 \pm 0.806$ & 0.870 \\
\hline
\end{tabular}

Influx: drugs were added to the inner chamber and monitored in the outer chamber to mimic the flux from the blood to the brain. Efflux: in the opposite direction of the influx, to mimic the flux from the brain to the blood. 
Thus, AcPAS did not seem to be a substrate of P-gp.

At the high concentration, the PAS NER was 1.05, which is far below 2.0. While it was possible that this may result from a partial P-gp saturation at the MDCK-MDR1 cell monolayer due to the high PAS concentration, it also seemed likely that PAS at the high concentration might have an inhibitory effect on P-gp-mediated efflux. To test this hypothesis, we conducted experiments to investigate whether PAS competitively inhibited R123 efflux transport in the MDCK-MDR1 cell monolayer. Data presented in Figure 4 showed that with the increase of PAS concentrations (0-400 $\mu \mathrm{g} / \mathrm{mL})$, the R123 efflux was proportionally reduced with a PAS $\mathrm{IC}_{50}$ of $10.3 \mu \mathrm{g} / \mathrm{mL}$. Moreover, when AcPAS $(2 \mu \mathrm{g} / \mathrm{mL}$ or $50.0 \mu \mathrm{g} / \mathrm{mL}$ in transport buffer) was studied in the same system, AcPAS did not exert any effect on the R123 NER (data not shown). These data suggest that PAS, but not AcPAS, appeared to be a P-gp inhibitor.

\section{Transport study of PAS and AcPAS in MDCK-MRP1 cells}

The MDCK-MRP1 cells were used to evaluate whether the PAS or AcPAS efflux at the BBB was mediated by MRP1. When the cultured cells reached confluency in the inner chamber, the TEER values were above $500 \Omega \cdot \mathrm{cm}^{2}$ and the $P_{\mathrm{E}}$ of phenol red was below $3.97 \times 10^{-8} \mathrm{~cm} / \mathrm{s}$. Similar to the results using the MDCK-MDR1 cells, the concentration-time curves of both compounds were linear in both the efflux and the influx studies up to $2 \mathrm{~h}$. The results in Table 3 show that the NER of calcein-AM, a known substrate of MRP1, was 21.39, which was significantly reduced to 7.48 when the cells were treated with the MRP inhibitor MK-571 (Table 3). The PAS and AcPAS NER transport across the MDCK-MRP1/MDCK-WT cell monolayer were greater than 1.5 , which were indicative of MRP1-mediated efflux. The presence of MK-571 (20 $\mu \mathrm{mol} / \mathrm{L})$ in the culture significantly reversed the PAS and AcPAS efflux through the MDCK-MRP1/MDCK-WT cell monolayer; the NER was reduced to almost 1.0. These data indicate that that the efflux of both PAS and AcPAS may be mediated by MRP1.

\section{Discussion}

The potential usefulness of PAS in the treatment of Mninduced parkinsonian disorders requires an in-depth understanding of the drug's pharmacokinetic characteristics in the $\mathrm{CNS}^{[21]}$. PAS has been proven to be an effective agent for the treatment of Mn-induced neurotoxicity in brain cells ${ }^{[20]}$, in experimental animals ${ }^{[13,16,18,19]}$, and in humans ${ }^{[11,12,14,15,17]}$. However, the mechanisms by which PAS and its metabolite AcPAS are transported across the BBB remained unknown. Our previous studies have shown that the AcPAS brain-toplasma ratios in the cerebellum, thalamus, motor cortex, hippocampus and striatum are significantly greater than the PAS ratios $^{[21]}$. The low PAS concentration in brain tissues could be due to the highly efficient clearance of PAS by an efflux transporter, such as P-gp, that pumps PAS back into the blood. The higher AcPAS concentration could be due to P-gp's unique preference for PAS over its metabolite. The present study used the MDR1a-null mouse model and provided the evidence to support this hypothesis.

Using the US FDA (2012) recommended MDCK-MDR1 and MDCK-MRP1 cell models, the current study further examined whether PAS and AcPAS were substrates of P-gp and/or MRP1. Our data clearly show that PAS is P-gp substrate at the BBB. First, in our in vivo studies with intravenous PAS administration, the brain-to-plasma ratios of PAS concentrations in the brain parenchyma and capillary were much greater in the MDR1a-null mice compared to wild-type mice; thus, a lack of P-gp removal of PAS resulted in the accumulation of PAS in the brain capillary as well as in brain tissues. Second, the NER of PAS at low concentrations transport across the MDCKMDR1/MDCK-WT cell monolayer was greater than 2.0, which falls into FDA's definition of P-gp-mediated transport. Lastly, the P-gp inhibitors significantly reversed the efflux effect of PAS in the MDCK-MDR1 cell monolayer. Thus, these in vivo and in vitro findings support the view that P-gp plays a crucial role in PAS distribution in the brain.

Table 3. Permeability $\left(P_{\mathrm{E}}\right)$ of PAS and AcPAS through the MDCK/MDCK-MRP1 cell monolayer in the Transwell devices. Data represent the mean \pm SD. $n=3$. ${ }^{\mathrm{b}} P<0.05$ compared to the group not given the MK-571.

\begin{tabular}{|c|c|c|c|c|c|c|c|}
\hline & \multirow{2}{*}{$\begin{array}{l}\text { Permeability } \\
\left(\times 10^{-7} \mathrm{~cm} / \mathrm{s}\right)\end{array}$} & & \multicolumn{2}{|c|}{ MDCK monolayer } & \multicolumn{2}{|c|}{ MDCK-MDR1 monolayer } & \multirow{2}{*}{$\begin{array}{l}\text { Net efflux } \\
\text { ratio (NER) }\end{array}$} \\
\hline & & & Influx & Efflux & Influx & Efflux & \\
\hline & Calcein-AM (4 $\mu \mathrm{mol} / \mathrm{L})$ & MK-571 (-) & $5.817 \pm 0.170$ & $4.521 \pm 0.595$ & $1.330 \pm 0.126$ & $22.11 \pm 3.999$ & 21.39 \\
\hline & & MK-571 (+) & $4.217 \pm 0.402$ & $4.162 \pm 0.450$ & $1.830 \pm 0.260$ & $13.51 \pm 1.599$ & $7.482^{b}$ \\
\hline \multirow[t]{4}{*}{ PAS } & High conc $(430 \mu \mathrm{g} / \mathrm{mL})$ & MK-571 (-) & $5.311 \pm 0.883$ & $4.083 \pm 0.462$ & $4.097 \pm 0.232$ & $6.560 \pm 0.606$ & 2.083 \\
\hline & & MK-571 (+) & $5.839 \pm 0.323$ & $5.668 \pm 0.532$ & $6.128 \pm 0.186$ & $5.766 \pm 0.298$ & $0.969^{b}$ \\
\hline & Low conc $(2 \mu \mathrm{g} / \mathrm{mL})$ & MK-571 (-) & $5.169 \pm 0.558$ & $2.721 \pm 0.145$ & $3.606 \pm 0.412$ & $8.267 \pm 1.079$ & 4.355 \\
\hline & & MK-571 (+) & $4.765 \pm 0.507$ & $3.766 \pm 0.362$ & $4.217 \pm 0.291$ & $4.939 \pm 0.288$ & $1.482^{b}$ \\
\hline \multirow[t]{4}{*}{ AcPAS } & High conc $(50 \mu \mathrm{g} / \mathrm{mL})$ & MK-571 (-) & $46.14 \pm 6.993$ & $37.32 \pm 7.350$ & $27.64 \pm 3.276$ & $35.39 \pm 4.998$ & 1.583 \\
\hline & & MK-571 (+) & $67.68 \pm 4.872$ & $63.63 \pm 7.791$ & $39.27 \pm 2.478$ & $32.95 \pm 4.242$ & $0.892^{b}$ \\
\hline & Low conc $(2 \mu \mathrm{g} / \mathrm{mL})$ & MK-571 (-) & $35.55 \pm 4.347$ & $32.93 \pm 2.835$ & $26.82 \pm 2.226$ & $43.83 \pm 5.523$ & 1.765 \\
\hline & & MK-571 (+) & $44.73 \pm 6.636$ & $61.91 \pm 12.92$ & $30.18 \pm 1.827$ & $41.18 \pm 3.885$ & $0.986^{b}$ \\
\hline
\end{tabular}


Not only does the current study indicate that PAS is a P-gp substrate, but it also suggests that PAS is a P-gp competitive inhibitor. In the MCDK-MDR1 cells, PAS produced a concentration-dependent inhibition of R123 efflux, indicative of P-gp inhibition at higher concentrations. This is consistent with our in vitro data that shows less efflux at the high PAS concentration. Our previous in vivo studies administering PAS (200 $\mathrm{mg} / \mathrm{kg}$, iv) to rats resulted in a rapid increase of PAS in brain parenchyma ${ }^{[21]}$; this could be ascribed to the PAS-induced inhibition of P-gp at the brain capillary endothelial cells. Data in the literature indicate that both the cerebellum and the thalamus possess abundant brain capillaries ${ }^{[36,37]}$; thus, the inhibition of P-gp at the capillaries could result rapidly upon the entrance of PAS into the cerebellum and thalamus with a $C_{\max }$ within $15 \mathrm{~min}^{[21]}$. The ratio of the steady-state maximum total plasma inhibitor concentration to the in vitro inhibitory potential $\left(\mathrm{IC}_{50}\right)$ is $1.94(>0.1)$, indicating a systemic P-gp-mediated drug-drug interaction in the clinic ${ }^{[38-41]}$. These observations suggest that for clinical uses of PAS to treat Mn neurotoxicity, the inhibitory effects of PAS at high-doses on P-gp should be taken into account.

Our results clearly demonstrate that AcPAS is not a P-gp substrate. The AcPAS $P_{\mathrm{E}}$ values also indicated that AcPAS had a greater permeability than PAS at the MDCK cell monolayer, apparently due to better passive diffusion. As a result, AcPAS may cross the BBB more easily than PAS with a higher $C_{\max }$ and $\mathrm{AUC}_{0-\infty}$ and a longer $t_{1 / 2}$ in most brain regions. Furthermore, based on our previous work ${ }^{[21]}$, AcPAS has a stronger tissue binding affinity with brain tissue protein. The higher tissue binding of AcPAS may allow the AcPAS to be eliminated by brain barrier systems and spinal cord more slowly than PAS. All of these reasons may explain our earlier observation that after PAS administration, only AcPAS, but not PAS, could be detected in the brain capillary fraction and that AcPAS was present at higher brain tissue concentrations than PAS in previous ${ }^{[21,33]}$ and in current studies. Whether PAS may be biotransformed to AcPAS in the brain requires further investigation.

Data from the literature suggest that $\mathrm{Mn}$ in blood can gain access to the CSF via the BCB in the choroid plexus and further accumulate in the striatum and hippocampus ${ }^{[18,42-47]}$. Our earlier study reported an effective reduction in Mn levels in the choroid plexus following PAS administration ${ }^{[18]}$. The most abundant efflux transporter in the BCB is MRP1 (ABCC1) $)^{[48]}$, which exists at the basolateral side of choroid plexus epithelium $^{[30]}$ and participates in maintaining CNS homeostasis by blocking access of circulatory neurotoxicants to the brain. The current studies in the Transwell transport devices with the MDCK-MRP1 cells showed that MRP1 regulated the extravasation of both PAS and AcPAS at the brain barrier and that this regulation could be inhibited by the MRP inhibitor MK-571. These in vitro findings are consistent with our in vivo observations that MK-571 injection increased the brain PAS concentration in MDR1a-null mice, and increased brain AcPAS concentrations in both wild-type and MDR1a-null mice. The in vivo data indicate that MRP is involved in the transport of both PAS and AcPAS in the brain. The results above suggest a possible pathway for both compounds to be effluxed by MRP to the blood from the CSF via the BCB route. While the current study provides valuable information on the transport of both PAS and AcPAS by MRP1, we cannot exclude the impact of other transporters at the BCB. Spector and Lorenzo suggested that PAS, in an artificial CSF, was favored for the efflux transport to blood rather than the influx by the organic acid transporter 3 (OAT-3) $)^{[49,50]}$. The pathway of these two potential chelators at the $\mathrm{BCB}$ may contribute to the effective reduction of $\mathrm{Mn}$ in the choroid plexus after PAS administration ${ }^{[18]}$.

Our results from the in vitro study, the in vivo animal model, and the pharmacokinetic study, suggest that after administration, transporters in the ABC family, including P-gp and MRP, have the capability to eliminate PAS from the ISF back to the blood (Figure 5A). AcPAS in blood can gain access to the CNS through the cerebral capillary endothelium, and accumulate in striatum, hippocampus and motor cortex mainly in the free, unbound form (Figure 5B).
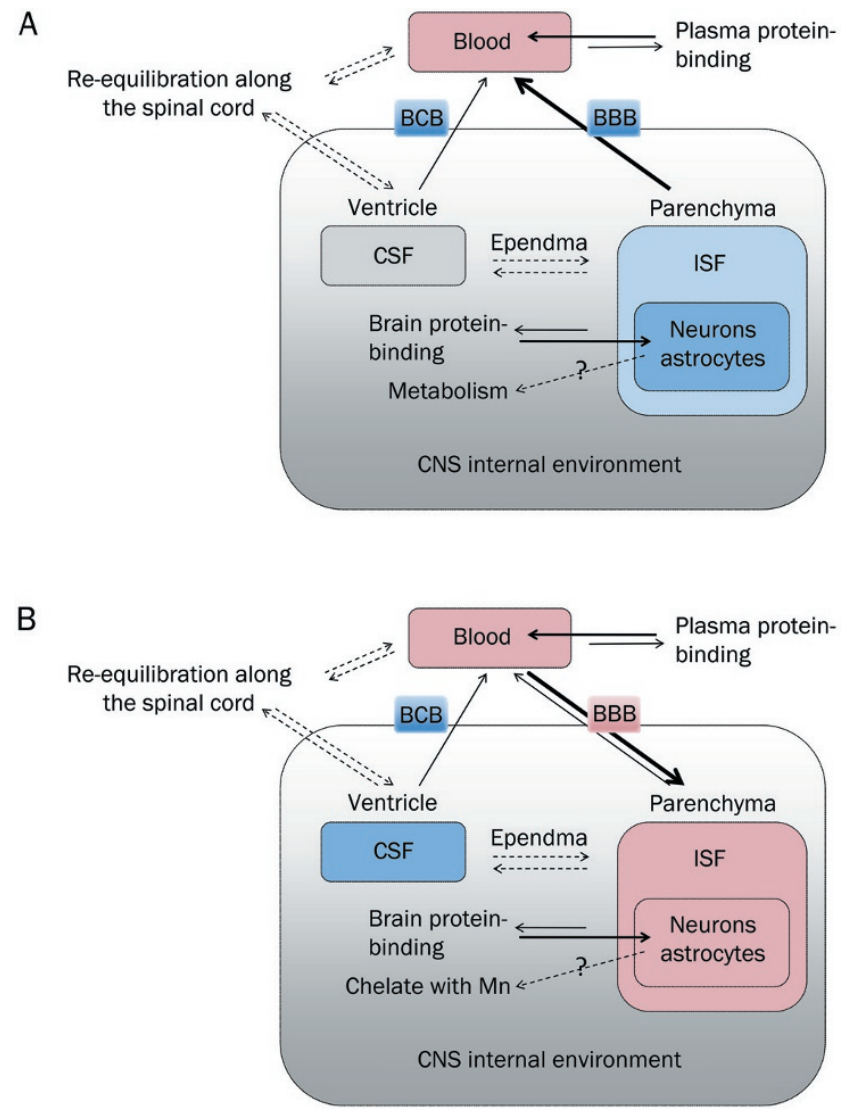

Figure 5. The possible disposition routes by which (A) PAS or (B) AcPAS remove $\mathrm{Mn}$ from the central nervous system (CNS) internal environment. Red areas represent the regions with relatively high concentrations of the compound, whereas blue areas represent the regions with relatively low concentrations. Thick arrows represent the major transport direction of the compound at the brain barrier systems. 


\section{Conclusion}

In the current study, we used MDR1a-null mice and MDCKMDR1 cells to examine PAS and AcPAS accumulation in the brain. Our results indicate that P-gp plays a significant role in the efflux transport of PAS from the brain parenchyma through the BBB back into blood. The in vitro studies using the MDCK-MRP1 cells also provide evidence that MRP1 is involved in both PAS and AcPAS transport in the brain. These findings are important for the clinical application of PAS in the treatment of Mn-induced neurotoxicity.

\section{Abbreviations}

AcPAS, N-acetyl-para-aminosalicylic acid; ANOVA, analysis of variance; $\mathrm{AUC}_{0-\infty}$, area under the concentration-time curve from time zero to infinity; $\mathrm{BBB}$, blood-brain barrier; $\mathrm{BCB}$, blood-cerebrospinal fluid barrier; $C_{\max }$ maximal plasma concentration after dosing; CNS, central nervous system; CSF, cerebrospinal fluid; ER, efflux ratio; HPLC, high-performance liquid chromatography; ISF, interstitial fluid; Mn, manganese; MTT, 3-(4,5-dimethyl-2-thiazolyl)-2,5-diphenyl-2H-tetrazolium bromide; MDR1: multidrug resistance protein 1; MRP1, multidrug resistance-associated protein 1; NER, net efflux ratio; PAS, 4-amino-2-hydroxybenzoic acid; $P_{\mathrm{E}}$, permeability; PBS, phosphate buffered solution; P-gp, P-glycoprotein; R123, rhodamine $123 ; t_{1 / 2}$, half-time; TEER, transepithelial electrical resistance.

\section{Acknowledgements}

This work was supported by the US National Institute of Health/National Institute of Environmental Health Grant RO1 ES008146 (Wei ZHENG), the US Department of Defense Contract USAMRMC W81XWH-05-1-0239 (Wei ZHENG), and the National Major Projects from the Ministry of Science and Technology of China (2012ZX09506001-004) (Su ZENG).

\section{Author contribution}

Lan HONG, Wei ZHENG, and Su ZENG participated in the research design; Lan HONG, Cong XU, Stefanie O'NEAL conducted the experiments; Hui-chang BI and Min HUANG contributed new reagents or analytic tools; Lan HONG, Cong XU, and Wei ZHENG performed the data analysis; Lan HONG, Wei ZHENG, Stefanie O'NEAL, and Su ZENG wrote or contributed to the writing of the manuscript.

\section{References}

1 Aschner M, Guilarte TR, Schneider JS, Zheng W. Manganese: recent advances in understanding its transport and neurotoxicity. Toxicol Appl Pharmacol 2007; 221: 131-47.

2 Bowler RM, Gysens S, Diamond E, Nakagawa S, Drezgic M, Roels HA. Manganese exposure: neuropsychological and neurological symptoms and effects in welders. Neurotoxicology 2006; 27: 315-26.

3 Crossgrove J, Zheng W. Manganese toxicity upon overexposure. NMR Biomed 2004; 17: 544-53.

4 Myers JE, Thompson ML, Ramushu S, Young T, Jeebhay MF, London $L$, et al. The nervous system effects of occupational exposure on workers in a South African manganese smelter. Neurotoxicology 2003; 24: 885-94.
5 Calne DB, Chu NS, Huang CC, Lu CS, Olanow W. Manganism and idiopathic parkinsonism: similarities and differences. Neurology 1994; 44: 1583-6.

6 Cook DG, Fahn S, Brait KA. Chronic manganese intoxication. Arch Neurol 1974; 30: 59-64.

7 Ono K, Komai K, Yamada M. Myoclonic involuntary movement associated with chronic manganese poisoning. J Neurol Sci 2002; 199: 93-6.

8 Seaton CL, Lasman J, Smith DR. The effects of CaNa2EDTA on brain lead mobilization in rodents determined using a stable lead isotope tracer. Toxicol Appl Pharmacol 1999; 159: 153-60.

9 Hernandez E, Discalzi G, Valentini C, Venturi F, Chio A, Carmellino $C$, et al. Follow-up of patients affected by manganese-induced Parkinsonism after treatment with CaNa2EDTA. Neurotoxicology 2006; 27: 333-9.

10 Mitnick C, Bayona J, Palacios E, Shin S, Furin J, Alcantara F, et al. Community-based therapy for multidrug-resistant tuberculosis in Lima, Peru. N Engl J Med 2003; 348: 119-28.

11 Ky SQ, Deng HS, Xie PY, Hu W. A report of two cases of chronic serious manganese poisoning treated with sodium para-aminosalicylic acid. Br J Ind Med 1992; 49: 66-9.

12 Jiang YM, Mo XA, Du FQ, Fu X, Zhu XY, Gao HY, et al. Effective treatment of manganese-induced occupational Parkinsonism with p-aminosalicylic acid: a case of 17-year follow-up study. J Occup Environ Med 2006; 48: 644-9.

13 Nelson M, Huggins T, Licorish R, Carroll MA, Catapane EJ. Effects of $p$-aminosalicylic acid on the neurotoxicity of manganese on the dopaminergic innervation of the cilia of the lateral cells of the gill of the bivalve mollusc, Crassostrea virginica. Comp Biochem Physiol C Toxicol Pharmacol 2010; 151: 264-70.

14 Shi XY. One case on chronic serious manganism treated with sodium para-aminosalicylic acid. J Baotou Med Coll 2002; 18: 161.

$15 \mathrm{Wu}$ P. A report of one case on the diagnostic treatment with PAS-Na. Ind Health Occup Dis 1991; 17: 163.

16 Yoon H, Kim DS, Lee GH, Kim JY, Kim DH, Kim KW, et al. Protective effects of sodium para-amino salicylate on manganese-induced neuronal death: the involvement of reactive oxygen species. J Pharm Pharmacol 2009; 61: 1563-9.

17 Zhao DF. The clinical observation on manganism treated with PAS-Na. J Guiyang Med Coll 1995; 20: 327.

18 Zheng W, Jiang YM, Zhang Y, Jiang W, Wang X, Cowan DM. Chelation therapy of manganese intoxication with para-aminosalicylic acid (PAS) in Sprague-Dawley rats. Neurotoxicology 2009; 30: 240-8.

19 Santos AP, Lucas RL, Andrade V, Mateus ML, Milatovic D, Aschner M, et al. Protective effects of ebselen (Ebs) and para-aminosalicylic acid (PAS) against manganese (Mn)-induced neurotoxicity. Toxicol Appl Pharmacol 2012; 258: 394-402.

20 Santos D, Batoreu MC, Aschner M, Marreilha dos Santos AP. Comparison between 5-aminosalicylic acid (5-ASA) and para-aminosalicylic acid (4-PAS) as potential protectors against Mn-induced neurotoxicity. Biol Trace Elem Res 2013; 152: 113-6.

21 Hong L, Jiang W, Pan H, Jiang Y, Zeng S, Zheng W. Brain regional pharmacokinetics of p-aminosalicylic acid and its $n$-acetylated metabolite: effectiveness in chelating brain manganese. Drug Metab Dispos 2011; 39: 1904-9.

22 Abbott NJ, Patabendige AA, Dolman DE, Yusof SR, Begley DJ. Structure and function of the blood-brain barrier. Neurobiol Dis 2010; 37: $13-25$.

23 Engelhardt B, Sorokin L. The blood-brain and the blood-cerebrospinal fluid barriers: function and dysfunction. Semin Immunopathol 2009; 31: 497-511. 
24 Zheng W, Aschner M, Ghersi-Egea JF. Brain barrier systems: a new frontier in metal neurotoxicological research. Toxicol Appl Pharmacol 2003; 192: 1-11.

25 Zheng W, Monnot AD. Regulation of brain iron and copper homeostasis by brain barrier systems: implication in neurodegenerative diseases. Pharmacol Ther 2012; 133: 177-88.

26 Daood M, Tsai C, Ahdab-Barmada M, Watchko JF. ABC transporter ( $\mathrm{P}$ gp/ABCB1, MRP1/ABCC1, BCRP/ABCG2) expression in the developing human CNS. Neuropediatrics 2008; 39: 211-8.

27 Baehr C, Reichel V, Fricker G. Choroid plexus epithelial monolayers-a cell culture model from porcine brain. Cerebrospinal Fluid Res 2006; 3: 13.

28 Klas J, Wolburg H, Terasaki T, Fricker G, Reichel V. Characterization of immortalized choroid plexus epithelial cell lines for studies of transport processes across the blood-cerebrospinal fluid barrier. Cerebrospinal Fluid Res 2010; 7: 11-22.

29 Loscher W, Potschka H. Role of drug efflux transporters in the brain for drug disposition and treatment of brain diseases. Prog Neurobiol 2005; 76: 22-76.

30 Spector R. Nature and consequences of mammalian brain and CSF efflux transporters: four decades of progress. J Neurochem 2010; 112: 13-23.

31 Sun H, Dai H, Shaik N, Elmquist WF. Drug efflux transporters in the CNS. Adv Drug Deliv Rev 2003; 55: 83-105.

32 USFDA (2012). Draft guidance for industry: drug interaction studiesstudy design, data analysis, and implications for dosing and labeling.

33 Hong L, Jiang W, Zeng S, Zheng W. HPLC analysis of para-aminosalicylic acid and its metabolite in plasma, cerebrospinal fluid and brain tissues. J Pharm Biomed Anal 2011; 54: 1101-9.

34 Deane R, Zheng W, Zlokovic BV. Brain capillary endothelium and choroid plexus epithelium regulate transport of transferrin-bound and free iron into the rat brain, J Neurochem 2004; 88: 813-20.

35 Liu Y, Yu CN, Zeng S. Establishment of madin-darby canine kidney cell line with high P-glycoprotein expression. Chin Pharm J 2009; 44: 1608-13.

36 Rhoton AL Jr. The cerebrum. Anatomy. Neurosurgery 2007; 61: $37-$ 118.

37 Schmahmann JD. Vascular syndromes of the thalamus. Stroke 2003; 34: 2264-78.

38 Huo X, Liu Q, Wang C, Meng Q, Sun H, Peng J, et al. Enhancement effect of P-gp inhibitors on the intestinal absorption and antiproliferative activity of bestatin. Eur J Pharm Sci 2013; 50: 420-8.

39 Wang L, Meng Q, Wang C, Liu Q, Peng J, Huo X, et al. Dioscin restores the activity of the anticancer agent adriamycin in multidrug-resistant human leukemia K562/adriamycin cells by down-regulating MDR1 via a mechanism involving NF-KB signaling inhibition. J Nat Prod 2013; 76: 909-14.

40 Huo X, Liu Q, Wang C, Meng Q, Sun H, Peng J, et al. Inhibitory effect of valsartan on the intestinal absorption and renal excretion of bestatin in rats. J Pharm Sci 2014; 103: 719-29.

41 Liu Q, Wang C, Meng Q, Huo X, Sun H, Peng J, et al. MDR1 and OAT1/OAT3 mediate the drug-drug interaction between puerarin and methotrexate. Pharm Res 2014; 31: 1120-32.

42 Bock NA, Paiva FF, Nascimento GC, Newman JD, Silva AC. Cerebrospinal fluid to brain transport of manganese in a non-human primate revealed by MRI. Brain Res 2008; 1198: 160-70.

43 Lai JC, Minski MJ, Chan AW, Leung TK, Lim L. Manganese mineral interactions in brain. Neurotoxicology 1999; 20: 433-44.

44 Li GJ, Choi BS, Wang X, Liu J, Waalkes MP, Zheng W. Molecular mechanism of distorted iron regulation in the blood-CSF barrier and regional blood-brain barrier following in vivo subchronic manganese exposure. Neurotoxicology 2006; 27: 737-44.

45 Reaney SH, Bench G, Smith DR. Brain accumulation and toxicity of $\mathrm{Mn}(\mathrm{II})$ and $\mathrm{Mn}$ (III) exposures. Toxicol Sci 2006; 93: 114-24.

46 Roels H, Meiers G, Delos M, Ortega I, Lauwerys R, Buchet JP, et al. Influence of the route of administration and the chemical form $\left(\mathrm{MnCl}_{2}\right.$, $\mathrm{MnO}_{2}$ ) on the absorption and cerebral distribution of manganese in rats. Arch Toxicol 1997; 71: 223-30.

47 Zheng W, Zhao Q, Slavkovich V, Aschner M, Graziano JH. Alteration of iron homeostasis following chronic exposure to manganese in rats. Brain Res 1999; 833: 125-32.

48 Redzic Z. Molecular biology of the blood-brain and the blood-cerebrospinal fluid barriers: similarities and differences. Fluids Barriers CNS 2011; 8: 3.

49 Spector R, Lorenzo AV. The active transport of para-aminosalicylic acid from the cerebrospinal fluid. J Pharmacol Exp Ther 1973; 185: 642-8.

50 Spector R, Lorenzo AV. The effects of salicylate and probenecid on the cerebrospinal fluid transport of penicillin, aminosalicyclic acid and iodide. J Pharmacol Exp Ther 1974; 188: 55-65. 